Pacific

Journal of

Mathematics

EXISTENCE AND NONEXISTENCE OF INTERIOR-PEAKED SOLUTION FOR A NONLINEAR NEUMANN PROBLEM

DaOmin CaO, E.S. Noussair, And Shusen Yan 


\title{
EXISTENCE AND NONEXISTENCE OF INTERIOR-PEAKED SOLUTION FOR A NONLINEAR NEUMANN PROBLEM
}

\author{
Daomin CaO, E.S. Noussair, and Shusen Yan
}

\section{We show that the critical problem}

$$
\left\{\begin{array}{l}
-\Delta u+\lambda u=u^{2^{*}-1}+a u^{q-1}, \quad u>0 \text { in } \Omega, \\
\frac{\partial u}{\partial \nu}=0 \text { on } \partial \Omega, \quad 2<q<2^{*}=2 N /(N-2),
\end{array}\right.
$$

has no positive solutions concentrating, as $\lambda \rightarrow \infty$, at interior points of $\Omega$ if $a=0$, but for a class of symmetric domains $\Omega$, the problem with $a>0$ has solutions concentrating at interior points of $\Omega$.

\section{Introduction.}

In this paper we consider the semilinear Neumann problem

$$
\left\{\begin{array}{l}
-\Delta u+\lambda u=|u|^{2^{*}-2} u+a|u|^{q-2} u \text { in } \Omega, \\
\frac{\partial u}{\partial \nu}=0 \text { on } \partial \Omega,
\end{array}\right.
$$

where $\Omega \subset \mathbb{R}^{N}(N \geq 3)$ is a bounded domain with smooth boundary having certain symmetries (to be specified below), $\lambda>0, a \geq 0$, are constants, $\nu$ is the unit outer normal to $\partial \Omega, 2^{*}=2 N /(N-2)$ and $2<q<2 N /(N-2)$.

Certain physical as well as biological situations have been modeled by such boundary value problems. We refer to [9], for such applications.

Much progress on the existence and qualitative behaviour of solutions for (1.1) with $a=0$ has been made in the last few years. To state the results, we define the energy functional associated with (1.1):

$$
I_{\lambda}(u)=\int_{\Omega} \frac{1}{2}\left(|\nabla u|^{2}+\lambda u^{2}\right)-\frac{1}{2^{*}} \int_{\Omega}|u|^{2^{*}}-\frac{a}{q} \int_{\Omega}|u|^{q}, u \in H^{1}(\Omega) .
$$

A solution of (1.1) is called a least energy solution if it minimizes the functional $I_{\lambda}(u)$ in $\left\{u \in H^{1}(\Omega):\left\langle I_{\lambda}^{\prime}(u), u\right\rangle=0, u \neq 0\right\}$. Of particular interest has been the existence of solutions which exhibit a concentration phenomenon when $\lambda \rightarrow \infty$. This was first shown for the problem

$$
\left\{\begin{array}{l}
-\Delta u+\lambda u=u^{p-1}, u>0 \text { in } \Omega, \\
\frac{\partial u}{\partial \nu}=0 \text { on } \partial \Omega,
\end{array}\right.
$$


in the subcritical case $2<p<2 N /(N-2)$, by Lin and $\mathrm{Ni}$ in [10], Lin, $\mathrm{Ni}$ and Takagi in $[\mathbf{1 1}], \mathrm{Ni}$ and Takagi in $[\mathbf{1 3}, \mathbf{1 4}]$, where it is shown that a nonconstant least energy solution exists for $\lambda$ sufficiently large, and that such a solution must have only one maximum point on $\bar{\Omega}$, which must be on the boundary $\partial \Omega$ and tends to the point of maximum mean curvature of the boundary as $\lambda$ goes to $\infty$.

Similar studies have also been carried out for (1.3) in the critical case $(p=2 N /(N-2))$. In particular, Adimurthi and Mancini [1], Adimurthi, Mancini and Yavada [2], Adimurthi, Pacella and Yavada [4], and Wang $[\mathbf{2 4}, \mathbf{2 6}]$ have established the existence and multiplicity of solutions. In [3] as well as in [27], the concentration behavior of such solutions is also established.

A solution of (1.1) is said to be a low-energy solution if its energy is less than $\frac{S^{N / 2}}{2 N}$, where $S$ is the Sobolev constant. It has been shown in [4], [15], that all such solutions share a common property, namely that they are single-peaked on $\partial \Omega$ in the sense that each solution attains its maximum on $\bar{\Omega}$, only at one point on $\partial \Omega$. In [2] and [20], among other results, the existence of solutions of (1.3) concentrating at a nondegenerate critical point of the mean curvature of $\partial \Omega$ as $\lambda$ goes to infinity has been established when $N \geq 6$. It has also been shown in [4], [27] for $N \geq 5$, that if $P_{0}$ is a strict local maximum point of the mean curvature function of the boundary $\partial \Omega$, then for $\lambda$ sufficiently large, there exists a nonconstant low energy solution of (1.3) with $p=2 N /(N-2)$, with its peaks $P_{\lambda} \in \partial \Omega$ converging to $P_{0}$ as $\lambda \rightarrow \infty$. See also [28] for a survey. The same result was proved in [21] for $N=3$.

In addition to the study of low energy solutions of (1.2) with $2<p \leq$ $2 N /(N-2)$, recently much effort has been made to study the existence and qualitative behaviour of higher energy solutions, see for example [20], $[\mathbf{2 5}],[\mathbf{2 6}],[\mathbf{2 7}],[\mathbf{2 9}]$. For the special case when the domain has certain symmetries, Wang in [25], [29], Maier-Paape, K. Schmitt, Z.-Q. Wang in [16], showed the existence of solutions of (1.3), $(p=2 N /(N-2))$; with multiple peaks (of related symmetry), located on $\partial \Omega$.

Recently Z.-Q. Wang [25] showed that if $\Omega=-\Omega, N=3,4$, then for $\lambda$ sufficiently large, there is a solution of (1.3), $p=2 N /(N-2)$, which concentrates at either two points on $\partial \Omega$ or only at the origin. Wang's result does not specify which of the two cases occurs.

To the best of the authors knowledge there does not seem to be any result on the existence of solutions of $(1.3), p=2 N /(N-2)$, with interior peaks. In the sub-critical case there are some results on the existence and location of interior peaks. See for example [8], [30], [31]. The purpose of this paper is to study the existence of positive solutions of (1.1) which concentrate at interior points of $\Omega$. 
One of our main results in this paper, Theorem 1.2 shows that if $a=0$ in (1.1), then there are no positive solutions with interior peaks. On the other hand, if $a>0$, we show, Theorem 1.1, that, under some symmetry conditions on $\Omega$, (1.1) has, for large $\lambda$, a positive solution $u_{\lambda}$ and $u_{\lambda}$ concentrates at an interior point of $\Omega$ as $\lambda$ goes to infinity.

To state our main results, let us first introduce some notations.

For the existence of an interior-peaked solution we shall make the following symmetric assumption on $\Omega$.

(S) There is a subgroup $G \subset O(N)$, the orthogonal group in $\mathbb{R}^{N}$, such that $\Omega$ is $G$ invariant, that is, $x \in \Omega$ if and only if $G x \in \Omega, 0 \in \Omega$, and for any $x \in \Omega \backslash\{0\}, \# G x \geq 3$. Here \#Gx denotes the cardinal number of $G x$, the $G$-orbit of $x$.

We approach the existence problem using variational methods. Let $H_{G}^{1}$ be the subspace of $H^{1}(\Omega)$, defined by

$$
H_{G}^{1}=\left\{u \in H^{1}(\Omega) \mid g u(x) \triangleq u(g(x))=u(x) \text { for all } g \in G\right\} .
$$

Let $I_{\lambda}(u)$ be defined by (1.2) for $u \in H_{G}^{1}$. It is not difficult to check that there exists $0 \not \equiv u_{0} \in H_{G}^{1}(\Omega)$ such that $u_{0} \geq 0$ and $I_{\lambda}\left(u_{0}\right)<0$.

Define

$$
\begin{aligned}
\Gamma & =\left\{\gamma \in C^{1}\left([0,1], H_{G}^{1}\right) \mid \gamma(0)=0, \gamma(1)=u_{0}\right\} \\
c_{\lambda} & =\inf _{\gamma \in \Gamma} \sup _{t \in[0,1]} I_{\lambda}(\gamma(t)) \\
S & =\inf \left\{\frac{\int_{\Omega}|\nabla u|^{2}}{\left(\int_{\Omega}|u|^{2 *}\right)^{2 / 2^{*}}} \mid u \in H^{1}(\Omega) \backslash\{0\}\right\} .
\end{aligned}
$$

It is known [17] that critical points of $I_{\lambda}$ in $H_{G}^{1}$ are also critical points of $I_{\lambda}$ in $H^{1}(\Omega)$, and hence classical solutions of (1.1) by standard regularity arguments. The main difficulty in applying variational methods in this case is the fact that $I_{\lambda}$ fails to satisfy the Palais-Smale condition. Our arguments for the existence proof are based on establishing that the value $c_{\lambda}$ of $I_{\lambda}$ in $H_{G}^{1}$ lies in $\left(-\infty, S^{N / 2} / N\right)$. In such an interval $I_{\lambda}$ satisfies the PalaisSmale condition. This will establish the existence of a solution $u_{\lambda}$, which is a "global" minimizer of $I_{\lambda}$ in $\left\{u \in H_{G}^{1} \mid\left\langle I_{\lambda}^{\prime}(u), u\right\rangle=0, u \neq 0\right\}$. Using concentration-compactness arguments and the symmetry condition $(\mathbf{S})$, we show that $I_{\lambda}\left(u_{\lambda}\right) \rightarrow \frac{S^{N / 2}}{N}$, as $\lambda \rightarrow \infty$, and that $u_{\lambda}$ concentrates at exactly one interior point of $\Omega$. More precisely, define for $x \in \mathbb{R}^{N}$

$$
U(x)=\frac{[N(N-2)]^{\frac{N-2}{4}}}{\left(1+|x|^{2}\right)^{\frac{N-2}{2}}}
$$


and for $\mu>0, y \in \mathbb{R}^{N}, u \in H^{1}\left(\mathbb{R}^{N}\right)$ set

$$
u_{\mu, y}(x)=\mu^{\frac{N-2}{2}} u(\mu(x-y)) .
$$

Our first main result is:

Theorem 1.1. Suppose $a>0, \Omega$ satisfies condition $(\mathbf{S})$ and $q \in\left(2,2^{*}\right)$ for $N \geq 4, q \in(4,6)$ for $N=3$. Then (1.1) has a positive solution $u_{\lambda}$ for $\lambda$ sufficiently large. Furthermore,

$$
\lim _{\lambda \rightarrow \infty}\left\|u_{\lambda}(x)-\mu_{\lambda}^{\frac{N-2}{2}} U\left(\mu_{\lambda} x\right)\right\|_{H^{1}(\Omega)}=0,
$$

where $\mu_{\lambda} \rightarrow \infty$, as $\lambda \rightarrow \infty$.

Our second main result is:

Theorem 1.2. Suppose $N \geq 5$. Then (1.1) with $a=0$ has no positive solution $u_{\lambda}$ such that for some integer $k \geq 1$

$$
\lim _{\lambda \rightarrow \infty}\left\|u_{\lambda}-\sum_{i=1}^{k} U_{\mu_{i, \lambda}, y_{\lambda}^{i}}\right\|_{H^{1}(\Omega)}=0,
$$

with $\mu_{i, \lambda} \rightarrow \infty, y_{\lambda}^{i} \in \Omega, y_{\lambda}^{i} \rightarrow y^{i} \in \Omega$, as $\lambda \rightarrow \infty$ and $y^{i} \neq y^{j}$ for $i, j=$ $1, \cdots, k, \quad i \neq j$.

This paper is organized as follows. In Section 2, by exploiting the symmetry of $\Omega$, we establish the existence of an interior-peaked positive solution of (1.1) with $a>0$, and prove Theorem 1.1. In Section 3 we prove Theorem 1.2 by a reduction procedure.

\section{Existence of interior-peaked positive solutions.}

In this section we give the proof of Theorem 1.1. This is accomplished by the following Lemmas.

Lemma 2.1. Let $u_{0}, c_{\lambda}$ be as in (1.5), (1.6). Then

(i) $c_{\lambda}$ does not depend on the choice of $u_{0}$.

(ii) $0<c_{\lambda}=\inf \left\{I_{\lambda}(u) \mid u \in H_{G}^{1} \backslash\{0\},\left\langle I_{\lambda}^{\prime}(u), u\right\rangle=0\right\}$.

Lemma 2.1 follows by the same argument as in W.M. Ni and I. Takagi $[13]$.

Lemma 2.2. Suppose $a>0$. Let $u_{0}, c_{\lambda}$ be as in (1.5), (1.6). Then

$$
0<c_{\lambda}<\frac{S^{N / 2}}{N}
$$

for $q \in\left(2,2^{*}\right)$ if $N \geq 4$ and for $q \in(4,6)$ if $N=3$. 
Proof. We only need to construct a suitable test function. Let

$$
w_{\epsilon}(x)=\frac{1}{\left(\epsilon+|x|^{2}\right)^{\frac{N-2}{2}}}, \epsilon>0 .
$$

Then, as in H. Brezis and L. Nirenberg [7], we have

$$
\sup _{t \geq 0} I_{\lambda}\left(t w_{\epsilon}\right)<\frac{S^{N / 2}}{N}
$$

provided $\epsilon$ is sufficiently small. The same argument in [7] then implies that $c_{\lambda}>0$ and $c_{\lambda}<\frac{S^{N / 2}}{N}$. This completes the proof of Lemma 2.2.

The following result can be established using arguments based on the concentration compactness principle of P.L. Lions [12], see also Struwe [22]. Similar results were given by Z-Q. Wang [25] and D. Pierotti and S. Terracini [18].

Lemma 2.3. Let $\lambda>0$ be fixed. Let $\left(u_{n}\right)_{n} \subset H^{1}(\Omega)$, be a Palais-Smale sequence for $I_{\lambda}$ at level $c\left((P . S)_{c}\right.$ sequence $)$, i.e., $I_{\lambda}\left(u_{n}\right) \rightarrow c$ and $I_{\lambda}^{\prime}\left(u_{n}\right) \rightarrow 0$, as $n \rightarrow \infty$. Then there exist integers $k_{1} \geq 0, k_{2} \geq 0$, a solution $u_{*}$ of (1.1), $x_{n}^{i} \in \bar{\Omega}$, and sequences $\left(\mu_{n}^{i}\right)_{n}, \mu_{n}^{i} \rightarrow \infty$, as $n \rightarrow \infty, i=1,2, \cdots, k_{1}+k_{2}$, such that, up to a subsequence,

$$
\begin{gathered}
u_{n} \rightarrow u_{*} \text { weakly in } H^{1}(\Omega), \text { as } n \rightarrow \infty, \\
\left\|u_{n}-u_{*}-\sum_{i=1}^{k_{1}+k_{2}} V_{\mu_{n}^{i}, x_{n}^{i}}^{i}\right\|_{H^{1}(\Omega)} \rightarrow 0, \text { as } n \rightarrow \infty, \\
c=I_{\lambda}\left(u_{*}\right)+\sum_{i=1}^{k_{1}}\left(\frac{1}{2} \int_{\mathbb{R}_{+}^{N}}\left|\nabla V^{i}\right|^{2}-\frac{1}{2^{*}} \int_{\mathbb{R}_{+}^{N}}\left|V^{i}\right|^{2^{*}}\right) \\
+\sum_{i=k_{1}+1}^{k_{1}+k_{2}}\left(\frac{1}{2} \int_{\mathbb{R}^{N}}\left|\nabla V^{i}\right|^{2}-\frac{1}{2^{*}} \int_{\mathbb{R}^{N}}\left|V^{i}\right|^{2^{*}}\right) .
\end{gathered}
$$

Furthermore, we have

$$
\mu_{n}^{i} d\left(x_{n}^{i}, \partial \Omega\right) \leq C<\infty, \quad i=1, \cdots, k_{1},
$$

and

$$
\mu_{n}^{i} d\left(x_{n}^{i}, \partial \Omega\right) \rightarrow \infty, \text { as } n \rightarrow \infty, \quad i=k_{1}+1, \cdots, k_{1}+k_{2} .
$$

For $i=1, \cdots, k_{1},\left|\nabla V^{i}\right| \in L^{2}\left(R_{+}^{N}\right)$, and $V^{i}$ is a nonzero solution of

$$
\begin{cases}-\Delta V=|V|^{2^{*}-2} V & x \in \mathbb{R}_{+}^{N}, \\ \frac{\partial V}{\partial x_{N}}=0 & x_{N}=0 .\end{cases}
$$

For $i=k_{1}+1, \ldots, k_{1}+k_{2},\left|\nabla V^{i}\right| \in L^{2}\left(R^{N}\right)$, and $V^{i}$ is a nonzero solution of

$$
-\Delta V=|V|^{2^{*}-2} V \quad x \in \mathbb{R}^{N} \text {. }
$$


Proof. By the assumptions on the sequence $u_{n}$, it is easy to show that $\left\{u_{n}\right\}$ is bounded in $H^{1}(\Omega)$. Thus there exist $u_{*} \in H_{G}$ such that by choosing a subsequence we have

$$
u_{n} \rightarrow u_{*} \quad \text { weakly in } H^{1}(\Omega) .
$$

Thus $u_{*}$ is a weak solution of (1.1). Let $v_{n}=u_{n}-u_{*}$, then $v_{n}$ is a (P.S) sequence of $I^{\infty}$ defined by

$$
I^{\infty}(v)=\frac{1}{2} \int_{\Omega}|\nabla v|^{2}-\frac{1}{2^{*}} \int_{\Omega}|v|^{2^{*}} .
$$

Furthermore, $I^{\infty}\left(v_{n}\right) \rightarrow c-I_{\lambda}\left(u_{*}\right)$. We can finish our proof by exactly the same argument used in the proof of Lemma 3.3 in [18] (taking $\delta \equiv 0$ in $[\mathbf{1 8}]$ ). For readers' convenience we give here a sketch in the following.

Let $B_{r}(x)$ denote the ball in $\mathbb{R}^{N}$ centered at $x$ with radius $r>0$. We will extend function $v \in H^{1}(\Omega)$ to $H^{1}\left(\mathbb{R}^{N}\right)$ by the extension operation $E$ such that

$$
\left.E(v)\right|_{\Omega}=v, \quad\|E(v)\|_{H^{1}\left(\mathbb{R}^{N}\right)} \leq C(\Omega)\|v\|_{H^{1}(\Omega)} .
$$

We will denote by the same symbol $v$ and its extension $E(v)$.

Let $D^{1,2}\left(\mathbb{R}^{N}\right)$ be the closure of the set of all the functions in $C_{0}^{\infty}\left(\mathbb{R}^{N}\right)$ with the norm $\|u\|=\|\nabla u\|_{L^{2}\left(\mathbb{R}^{N}\right)}$.

By applying the concentration compactness principle there exist $\mu_{n}>$ $0, x_{n} \in \bar{\Omega}, \tau>0$ such that

$$
\widetilde{v_{n}}=\mu_{n}^{\frac{2-N}{2}} v_{n}\left(\frac{x}{\mu_{n}}+x_{n}\right),
$$

satisfies

$$
\sup \left\{\int_{B_{1}(x)}\left|\nabla \widetilde{v_{n}}\right|^{2} \mid \frac{x}{\mu_{n}}+x_{n} \in \Omega\right\}=\int_{B_{1}(0)}\left|\nabla \widetilde{v_{n}}\right|^{2}=\tau .
$$

Let $\widetilde{\Omega_{n}}=\left\{x \in \mathbb{R}^{N} \mid \frac{x}{\mu_{n}}+x_{n} \in \Omega\right\}$. We then show that $\widetilde{v_{n}}$ converges to $V$ weakly in $D^{1,2}\left(\mathbb{R}^{N}\right)$ and in $H_{\text {loc }}^{1}\left(\mathbb{R}^{N}\right)$. As in [22] and [18] we can prove that the convergence actually holds in the strong $H_{\text {loc }}^{1}\left(\mathbb{R}^{N}\right)$ sense. Thus, $V$ is not identically zero. From the fact that $v_{n}$ converge weakly to zero we get $\mu_{n} \rightarrow \infty$. Hence, we have the following two cases:

(1) $\mu_{n} \operatorname{dist}\left(x_{n}, \partial \Omega\right)$ goes to $\infty$,

(2) $\mu_{n} \operatorname{dist}\left(x_{n}, \partial \Omega\right)$ is bounded.

In the first case, $\widetilde{\Omega_{n}}$ tends to $\mathbb{R}^{N}$ and $V$ solves (2.6). In case (2), $\widetilde{\Omega_{n}}$ converges to a half space in the following sense: Up to a subsequence we can assume that the $x_{n}$ 's converge to $x_{0} \in \partial \Omega$; moreover if $y_{n}$ realizes the minimal distance of $x_{n}$ from $\partial \Omega$, we can assume that $\mu_{n}\left(y_{n}-x_{n}\right) \rightarrow z_{0}$. Let $W$ be a neighborhood of $x_{0}$ and $\Phi: U \subset \mathbb{R}^{N} \hookrightarrow W$ be a diffeomorphism such that $\Phi\left(U \cap \mathbb{R}_{+}^{N}\right)=W \cap \Omega$ and $\Phi\left(U \cap \mathbb{R}^{N-1}\right)=W \cap \partial \Omega$, and let $\xi_{n}=\Phi^{-1}\left(y_{n}\right) \rightarrow \xi_{0}=\Phi^{-1}\left(x_{0}\right)$. Define $\Phi_{n}(\xi)=\mu_{n}\left[\Phi\left(\frac{\xi}{\mu_{n}}+\xi_{n}\right)-x_{n}\right]$ : The 
$\Phi_{n}$ 's are charts for $\widetilde{\Omega_{n}}$ and converges $C^{1}$ on compact subsets of $\mathbb{R}^{N}$ to linear differmorohism $z_{0}+d \Phi\left(\xi_{0}\right)$. In this sense the boundaries $\partial \widetilde{\Omega_{n}}$ converge to the hyperplane $\left(z-z_{0}\right) \cdot \nu\left(x_{0}\right)=0$. Of course we can change our coordinate system over $\mathbb{R}^{N}$ in such a way that $z_{0}=0$ and $\nu\left(x_{0}\right)=-\mathbf{e}_{N}$. With this convention we conclude that $V$ solves (2.5). We can finish our proof by iterating the above procedure.

Remark 2.4. Notice that,

$$
\begin{array}{lc}
\frac{1}{2} \int_{\mathbb{R}_{+}^{N}}\left|\nabla V^{i}\right|^{2}-\frac{1}{2^{*}} \int_{\mathbb{R}_{+}^{N}}\left|V^{i}\right|^{2^{*}} \geq \frac{1}{2 N} S^{N / 2} \quad \text { for } i=1, \cdots, k_{1} \\
\frac{1}{2} \int_{\mathbb{R}^{N}}\left|\nabla V^{i}\right|^{2}-\frac{1}{2^{*}} \int_{\mathbb{R}^{N}}\left|V^{i}\right|^{2^{*}} \geq \frac{1}{N} S^{N / 2} & \text { for } i=k_{1}+1, \cdots, k_{1}+k_{2} .
\end{array}
$$

Remark 2.5. Notice that, by $(2.2), u_{n}-u_{*}$ concentrates, as $n \rightarrow \infty$, at the points $x_{n}^{i}, i=1, \cdots, k_{1}+k_{2}$. By (2.4) the points $x_{n}^{i}, i=1, \cdots, k_{1}$, approach $\partial \Omega$. It then follows from $u_{n}-u_{*} \in H_{G}^{1}$, and condition $(\mathbf{S})$, that either $k_{1}=0$ or $k_{1} \geq 3$.

Lemma 2.6. $I_{\lambda}$ satisfies the $(P . S)_{c}$ condition in $H_{G}^{1}(\Omega)$ for $c \in(-\infty$, $\left.S^{N / 2} / N\right)$.

Proof. Suppose $\left\{u_{n}\right\} \subset H_{G}^{1}(\Omega), u_{n} \geq 0$, is a (P.S $)_{c}$ sequence, with $c<\frac{S^{N / 2}}{N}$. It follows from Lemma 2.3 that there are integers $k_{1}, k_{2} \geq 0$, a solution $u_{*}$ of (1.1), and sequences $x_{n}^{i} \in \bar{\Omega}$ and $\mu_{n}^{i} \rightarrow \infty$, such that (2.2)-(2.4) hold. We first notice that $k_{2}=0$ since, otherwise, $c \geq \frac{S^{N / 2}}{N}$, contradicting our hypothesis on $c$. Notice also (see Remark 2.4) that $k_{1}=0$ since, otherwise $k_{1} \geq 3$, which implies that $c \geq \frac{3 S^{N / 2}}{2 N}$ contradicting our hypothesis on $c$. It then follows from (2.2) that $u_{n} \rightarrow u_{*}$ strongly in $H^{1}(\Omega)$, as $n \rightarrow \infty$. This completes the proof of Lemma 2.6.

Lemma 2.7. Assume $\Omega$ satisfies condition $(\mathbf{S})$ and $q \in\left(2,2^{*}\right)$ for $N \geq 4$, $q \in(4,6)$ for $N=3$. Then $c_{\lambda} \rightarrow \frac{S^{N / 2}}{N}$, as $\lambda \rightarrow \infty$.

Proof. To prove Lemma 2.7, we only need to show, by Lemma 2.2, that

$$
\lim _{\lambda \rightarrow \infty} c_{\lambda} \geq \frac{S^{N / 2}}{N}
$$

We argue by contradiction. Suppose that we have a sequence $\lambda_{n} \rightarrow \infty$, as $n \rightarrow \infty$, such that

$$
c_{\lambda_{n}} \rightarrow c<\frac{S^{N / 2}}{N}
$$

It follows from Lemma 2.2 and Lemma 2.6 that for each $\lambda_{n}$ ( $n$ large enough) we have a sequence $\left\{u_{n}\right\} \subset H_{G}^{1}(\Omega)$ satisfying

$$
I_{\lambda_{n}}\left(u_{n}\right)=c_{\lambda_{n}} \rightarrow c<\frac{S^{N / 2}}{N} .
$$


On the other hand, we have $I_{\lambda_{n}}\left(\left|u_{n}\right|\right)=I_{\lambda_{n}}\left(u_{n}\right)$, which means that $\left|u_{n}\right|$ achieves $\inf \left\{I_{\lambda_{n}}(u): u \in H_{G}^{1} \backslash\{0\},\left\langle I_{\lambda_{n}}^{\prime}(u), u\right\rangle=0\right\}$. By Langrange principle we have $I^{\prime}\left(u_{n}\right)=0$. Thus, without loss of generality we can assume that $u_{n} \geq 0, u_{n} \neq 0$ and satisfies

$$
\begin{cases}-\Delta u_{n}+\lambda_{n} u_{n}=u_{n}^{2^{*}-1}+a u_{n}^{q-1} & \text { in } \Omega, \\ \frac{\partial u_{n}}{\partial \nu}=0 & \text { on } \partial \Omega .\end{cases}
$$

By the standard regularity procedure and maximum principle we have $u_{n}>$ 0 in $\Omega$. It follows from (2.8), (2.9), and the Hölder's inequality, that

$$
0<\ell_{1}<\int_{\Omega}\left|\nabla u_{n}\right|^{2}+\lambda_{n} u_{n}^{2}<\ell_{2},
$$

uniformly for $n$, where $\ell_{1}$ and $\ell_{2}$ are some positive constants, independent of $n$. Hence by passing to a subsequence if necessary, we may assume that $u_{n} \rightarrow 0$ weakly in $H^{1}(\Omega)$, and by the compact embedding of $H^{1}(\Omega) \hookrightarrow$ $L^{2}(\Omega), \int_{\Omega} u_{n}^{2} \rightarrow 0$ as $\lambda_{n} \rightarrow \infty$. Set

$$
v_{n}(x)=\lambda_{n}^{-\frac{(N-2)}{4}} u_{n}\left(\frac{x}{\sqrt{\lambda_{n}}}\right), x \in \Omega_{n} \triangleq\left\{x \mid \frac{x}{\sqrt{\lambda_{n}}} \in \Omega\right\} .
$$

Then $v_{n}(x)$ satisfies

$$
\begin{cases}-\Delta v_{n}+v_{n}=v_{n}^{2^{*}-1}+\frac{a}{\lambda_{n}^{\sigma}} v_{n}^{q-1} & \text { in } \Omega_{n} \\ v_{n}>0 & \text { in } \Omega_{n} \\ \frac{\partial v_{n}}{\partial \nu}=0 & \text { on } \partial \Omega_{n}\end{cases}
$$

where

$$
\sigma=\frac{2^{*}-q}{4}(N-2)>0
$$

We also have

$$
c_{\lambda_{n}}=\frac{1}{2} \int_{\Omega_{n}}\left|\nabla v_{n}\right|^{2}+v_{n}^{2}-\frac{1}{2^{*}} \int_{\Omega_{n}}\left|v_{n}\right|^{2^{*}}-\frac{a}{q \lambda_{n}^{\sigma}} \int_{\Omega_{n}}\left|v_{n}\right|^{q} .
$$

Set

$$
\psi_{n}(x)=\chi_{\Omega_{n}}\left|v_{n}\right|^{2^{*}}
$$

where $\chi_{\Omega_{n}}$ is the characteristic function of $\Omega_{n}$. Then $\int_{\mathbb{R}^{N}} \psi_{n}(x) d x=$ $\int_{\Omega_{n}}\left|v_{n}\right|^{2^{*}}$. Without loss of generality, we may assume that $\int_{\Omega_{n}}\left|v_{n}\right|^{2^{*}} \rightarrow$ $\ell>0$, as $n \rightarrow \infty$, by (2.10). We now apply the concentration-compactness principle of P.L. Lions [12] to $\psi_{n}(x)$. We then have that one of the following three cases must occur:

(i) vanishing

(ii) compactness

(iii) dichotomy. 
If (i) occurs, then, by a result of Z.-Q. Wang, Lemma 4.6 in [26], we have $\int_{\Omega_{n}}\left|v_{n}\right|^{2^{*}} \rightarrow 0$, as $n \rightarrow \infty$, which leads to $\int_{\Omega_{n}}\left|\nabla v_{n}\right|^{2}+v_{n}^{2} \rightarrow 0$, as $n \rightarrow \infty$, contradicting (2.10). Thus (i) is excluded. As in Z.-Q. Wang [27] (iii) implies $c_{\lambda_{n}} \geq \frac{3 S^{N / 2}}{2 N}+o(1)$, since $v_{\lambda_{n}} \in H_{G}^{1}\left(\Omega_{n}\right)$. This contradicts (2.8).

We show next that (ii) cannot occur. Suppose (ii) occurs, that is, there is a sequence $\left\{y_{n}\right\}, y_{n} \in \Omega_{n}$ such that for all $\epsilon>0$, there is $R_{\epsilon}>0$, satisfying

$$
\int_{B_{R}\left(y_{n}\right) \cap \Omega_{n}}\left|v_{n}\right|^{2^{*}} \geq \ell-\epsilon
$$

for all $R \geq R_{\epsilon}$.

We claim that $\left\{y_{n}\right\}$ is bounded. In fact, since $v_{n} \in H_{G}^{1}\left(\Omega_{n}\right)$, if $\left|y_{n}\right| \rightarrow \infty$, as $n \rightarrow \infty$, we get a contradiction to (2.13). Hence suppose $y_{n} \rightarrow y_{0}$.

Set

$$
\mu_{n}=\left(\max _{x \in \bar{\Omega}} v_{n}(x)\right)^{4 /(N-2)}
$$

We claim that

$$
\lim _{n \rightarrow \infty} \mu_{n}=\infty
$$

To prove (2.14), suppose, to the contrary, that $\left\{\mu_{n}\right\}$ is bounded. Since $\int_{\Omega_{n}}\left|\nabla v_{n}\right|^{2}+\left|v_{n}\right|^{2}=\int_{\Omega}\left|\nabla u_{n}\right|^{2}+\lambda_{n} u_{n}^{2}$, is bounded by (2.10), we may assume that

$$
v_{n} \rightarrow v_{0} \quad \text { weakly in } H^{1}\left(\mathbb{R}^{N}\right) .
$$

Hence

$$
\begin{aligned}
\int_{B_{R}\left(y_{n}\right) \cap \Omega_{n}} v_{n}^{2} & \geq \frac{1}{\mu_{n}^{\left(\frac{N-2}{4}\right)\left(2^{*}-2\right)}} \int_{B_{R}\left(y_{n}\right) \cap \Omega_{n}} v_{n}^{2^{*}} \\
& =\frac{1}{\mu_{n}} \int_{B_{r}\left(y_{n}\right) \cap \Omega_{n}} v_{n}^{2^{*}} \geq \frac{1}{\mu_{n}}(\ell-\epsilon) .
\end{aligned}
$$

On the other hand,

$$
\int_{B_{2 R}\left(y_{0}\right)} v_{0}^{2} \geq \lim _{n \rightarrow \infty} \int_{B_{R}\left(y_{n}\right) \cap \Omega} v_{n}^{2} \geq \alpha>0
$$

by (2.16), for some $\alpha>0$, which implies that $v_{0} \geq 0, v_{0} \not \equiv 0$. But $v_{0}$ solves

$$
\begin{cases}-\Delta v_{0}+v_{0}=v_{0}^{2^{*}-1} & \text { in } \mathbb{R}^{N} \\ v_{0}>0 & \text { in } \mathbb{R}^{N},\end{cases}
$$


by (2.11), which is impossible, since the above equation has no solution. This proves (2.14). Set

$$
\begin{aligned}
w_{n}(x) & =\mu_{n}^{-\frac{(N-2)}{4}} v_{n}\left(\frac{x}{\sqrt{\mu_{n}}}\right), \quad x \in \sqrt{\mu_{n}} \Omega_{n}, \\
\tilde{\Omega}_{n} & \triangleq \sqrt{\mu_{n}} \Omega_{n}=\sqrt{\mu_{n} \lambda_{n}} \Omega .
\end{aligned}
$$

Then $w_{n}$ satisfies

$$
\begin{cases}-\Delta w_{n}+\frac{1}{\mu_{n}} w_{n}=w_{n}^{2^{*}-1}+\frac{a}{\lambda_{n}^{\sigma} \mu_{n}^{\sigma}} w_{n}^{q-1} & \text { in } \tilde{\Omega}_{n}, \\ w_{n}>0 & \text { in } \tilde{\Omega}_{n}, \\ \frac{\partial w_{n}}{\partial \nu}=0, & \text { on } \partial \tilde{\Omega}_{n}\end{cases}
$$

and

$$
\begin{aligned}
\int_{\tilde{\Omega}_{n}}\left|\nabla w_{n}\right|^{2} & =\int_{\Omega_{n}}\left|\nabla v_{n}\right|^{2} \\
\int_{\tilde{\Omega}_{n}}\left|w_{n}\right|^{2^{*}} & =\int_{\Omega_{n}}\left|v_{n}\right|^{2^{*}} .
\end{aligned}
$$

By standard elliptic regularity arguments, we have

$$
\begin{aligned}
& w_{n} \in C^{2, \gamma}(D), \\
& w_{n} \rightarrow w_{0} \quad \text { in } C_{\mathrm{loc}}^{2}\left(\mathbb{R}^{N}\right),
\end{aligned}
$$

for any compact subset $D \subset \mathbb{R}^{N}$. Hence $w_{0}$ satisfies

$$
\left\{\begin{array}{l}
-\Delta w_{0}=w_{0}^{2^{*}-1} \quad \text { in } \mathbb{R}^{N} \\
\left|\nabla w_{0}\right| \in L^{2}\left(\mathbb{R}^{N}\right) \\
w_{0} \in L^{2^{*}}\left(\mathbb{R}^{N}\right) \\
\max _{x \in \mathbb{R}^{N}} w_{0}(x)=1
\end{array}\right.
$$

By the uniqueness of solutions of the above problem, we conclude that $w_{0}(x)=\mu_{0}^{\frac{N-2}{4}} U\left(\sqrt{\mu_{0}}(x-y)\right)$ for some $\mu_{0}>0, y \in \mathbb{R}^{N}$, such that $w_{0}(y)=$ $\max _{x \in \mathbb{R}^{N}} w_{0}(x)$. Furthermore, we have

$$
\begin{aligned}
\int_{\mathbb{R}^{N}}\left|\nabla w_{0}\right|^{2} & =S^{N / 2}, \\
\int_{\mathbb{R}^{N}} w_{0}^{2^{*}} & =S^{N / 2} .
\end{aligned}
$$

Therefore, from $\int_{\mathbb{R}^{N}}\left|\nabla w_{0}\right|^{2} \leq \underline{\lim }_{n \rightarrow \infty} \int_{\tilde{\Omega}_{n}}\left|\nabla w_{n}\right|^{2}$, we obtain

$$
\underline{\lim }_{n \rightarrow \infty} \int_{\tilde{\Omega}_{n}}\left|\nabla w_{n}\right|^{2} \geq S^{N / 2}
$$


and

$$
\underline{\lim }_{n \rightarrow \infty}\left(\frac{1}{2} \int_{\tilde{\Omega}_{n}}\left|\nabla w_{n}\right|^{2}+\frac{1}{\mu_{n}} w_{n}^{2}-\frac{1}{2^{*}} \int_{\tilde{\Omega}_{n}} w_{n}^{2^{*}}-\frac{a}{q \lambda_{n}^{\sigma} \mu_{n}^{\sigma}} \int_{\tilde{\Omega}_{n}} w_{n}^{q}\right) \geq \frac{S^{N / 2}}{N},
$$

since

$$
\frac{1}{\lambda_{n}^{\sigma} \mu_{n}^{\sigma}} \int_{\tilde{\Omega}_{n}} w_{n}^{q} \leq \frac{1}{\lambda_{n}^{\sigma}}\left(\frac{1}{2 \mu_{n}} \int_{\tilde{\Omega}_{n}} w_{n}^{2}+\frac{1}{2} \int_{\tilde{\Omega}_{n}} w_{n}^{2^{*}}\right)
$$

by the Hölder's inequality.

But (2.19) contradicts (2.8), and we conclude that compactness cannot occur. Therefore (2.7) follows. This completes proof of Lemma 2.7.

\section{Proof of Theorem 1.1.}

Using Lemmas 2.2 and 2.6 we can obtain the existence of positive solution $u_{\lambda} \in H_{G}^{1}$. To establish (1.9), we follow the argument in Lemma 2.7 to show that only compactness can occur. By arguments very similar to that in the proof of Lemma 2.7 we show that there exists a sequence $\left\{\tilde{\mu}_{\lambda}\right\}, \tilde{\mu}_{\lambda} \rightarrow \infty$, such that

$$
\tilde{\mu}_{\lambda}^{-\frac{N-2}{4}} u_{\lambda}\left(\frac{x}{\sqrt{\tilde{\mu}_{\lambda}}}\right) \rightarrow w_{0}=\mu_{0}^{N-2} U\left(\sqrt{\mu_{0}}(x-y)\right), \quad \text { as } \lambda \rightarrow \infty,
$$

in $C_{\text {loc }}^{2}\left(\mathbb{R}^{N}\right)$, for some constant $\mu_{0}>0, y \in \mathbb{R}^{n}$, and

$$
\left\|\tilde{\mu}_{\lambda}^{-\frac{(N-2)}{4}} u_{\lambda}\left(\frac{x}{\sqrt{\tilde{\mu}_{\lambda}}}\right)-\mu_{0}^{\frac{N-2}{4}} U\left(\sqrt{\mu_{0}}(x-y)\right)\right\|_{H^{1}\left(\Omega_{\lambda}\right)} \rightarrow 0,
$$

as $\lambda \rightarrow \infty$, where $\Omega_{\lambda}=\frac{1}{\sqrt{\tilde{\mu}_{\lambda}}} \Omega$.

By the symmetry assumption, we have $y=0$, and

$$
\left\|u_{\lambda}(x)-\left(\mu_{0} \tilde{\mu}_{\lambda}\right)^{\frac{N-2}{4}} U\left(\sqrt{\mu_{0} \tilde{\mu}_{\lambda}} x\right)\right\|_{H^{1}(\Omega)} \rightarrow 0,
$$

as $\lambda \rightarrow \infty$. This completes the proof of Theorem 1.1.

\section{Nonexistence results.}

In this section we show that (1.1) with $a=0$ does not have solutions concentrating, as $\lambda \rightarrow \infty$, at interior points of $\Omega$.

Consider the problem

$$
\left\{\begin{array}{l}
-\Delta u+\lambda u=u^{2^{*}-1}, \quad u>0 \text { in } \Omega \\
\frac{\partial u}{\partial \nu}=0 \text { in } \partial \Omega
\end{array}\right.
$$

Let $k=1,2, \cdots$, be fixed. 
Define for $\mu=\left(\mu_{1}, \cdots, \mu_{k}\right) \in \mathbb{R}_{+}^{k}, y=\left(y^{1}, \cdots, y^{k}\right) \in \Omega^{k}$,

$$
\begin{aligned}
E_{\mu, y}^{k}= & \left\{w \in H^{1}(\Omega) \mid\left\langle U_{\mu_{i}, y^{i}}, w\right\rangle_{\lambda}=0,\right. \\
& \left\langle\frac{\partial U_{\mu_{i}, y^{i}}}{\partial \mu_{i}}, w\right\rangle_{\lambda}=0 \\
& \left\langle\frac{\partial U_{\mu_{i}, y^{i}}}{\partial y_{\ell}^{i}}, w\right\rangle_{\lambda}=0, \\
& i=1,2, \cdots, k ; \quad \ell=1,2, \cdots, N\},
\end{aligned}
$$

where $\langle u, v\rangle_{\lambda}=\int_{\Omega} \nabla u \nabla v+\lambda u v$. For $\delta>0$, define

$$
\begin{aligned}
M_{\delta}^{k}=\{ & (\alpha, \mu, y, \omega): \alpha=\left(\alpha_{1}, \cdots, \alpha_{k}\right) \in \mathbb{R}_{+}^{k}, \mu=\left(\mu_{1}, \cdots, \mu_{k}\right) \in \mathbb{R}_{+}^{k} . \\
& y=\left(y^{1}, \cdots, y^{k}\right) \in \Omega^{k}, \omega \in E_{\mu, y}^{k} \\
& \left|\alpha_{i}-1\right|<\delta, \mu_{i}>\frac{1}{\delta}, \operatorname{dist}\left(y^{i}, \partial \Omega\right)>\delta \\
& \text { for } i=1, \cdots, k,\|\omega\|<\delta\}
\end{aligned}
$$

In this section we will denote

$$
\begin{aligned}
\|u\|^{2} & =\int_{\Omega}|\nabla u|^{2}+u^{2} \quad \text { for } \quad u \in H^{1}(\Omega), \\
\|u\|_{q}^{q} & =\int_{\Omega}|u|^{q} \quad \text { for } \quad q \geq 1, u \in L^{q}(\Omega) .
\end{aligned}
$$

Let for $(\alpha, \mu, y, \omega) \in M_{\delta}^{k}, L_{\lambda}$ be defined by

$$
L_{\lambda}(\alpha, \mu, y, \omega)=I_{\lambda}\left(\sum_{i=1}^{k} \alpha_{i} U_{\mu_{i}, y^{i}}+\omega\right) .
$$

It is now well-known (see Bahri [5]) that if $\delta>0$ is small enough, then for $(\alpha, \mu, y, \omega) \in M_{\delta}^{k}, u=\sum_{i=1}^{k} \alpha_{i} U_{\mu_{i}, y^{i}}+\omega$ is a critical point of $I_{\lambda}(u)$ in $H^{1}(\Omega)$ if and only if $(\alpha, \mu, y, \omega)$ is a critical point of $L_{\lambda}(\alpha, \mu, y, \omega)$ in $M_{\delta}^{k}$, which is equivalent to the existence of $a^{i} \in \mathbb{R}, b^{i} \in \mathbb{R}, c_{\ell}^{i} \in \mathbb{R}$ for $i=1, \cdots, k$, 
$\ell=1, \cdots, N$, such that the following equations hold:

$$
\frac{\partial I_{\lambda}\left(\sum_{i=1}^{k} \alpha_{i} U_{\mu_{i}, y^{i}}+\omega\right)}{\partial \alpha_{i}}=0, \quad i=1, \cdots, k,
$$

$$
\begin{aligned}
\frac{\partial I_{\lambda}\left(\sum_{i=1}^{k} \alpha_{i} U_{\mu_{i} y^{i}}+\omega\right)(\phi)}{\partial \omega}= & \sum_{i=1}^{k}\left\{a^{i}\left\langle U_{\mu_{i}, y^{i}}, \varphi\right\rangle_{\lambda}+b^{i}\left\langle\frac{\partial U_{\mu_{i}, y^{i}}}{\partial \mu_{i}}, \varphi\right\rangle_{\lambda}\right. \\
& \left.+\sum_{\ell=1}^{N} c_{\ell}^{i}\left\langle\frac{\partial U_{\mu_{i}, y^{i}}}{\partial y_{\ell}^{i}}, \varphi\right\rangle_{\lambda}\right\}, \forall \varphi \in H^{1}(\Omega),
\end{aligned}
$$

$$
\frac{\partial I_{\lambda}\left(\sum_{i=1}^{k} \alpha_{i} U_{\mu_{i}, y^{i}}+\omega\right)}{\partial y_{j}^{i}}=b^{i}\left\langle\frac{\partial^{2} U_{\mu_{i}, y^{i}}}{\partial \mu_{i} \partial y_{j}^{i}}, \omega\right\rangle_{\lambda}+\sum_{\ell=1}^{N} c_{\ell}^{i}\left\langle\frac{\partial^{2} U_{\mu_{i}, y^{i}}}{\partial y_{\ell}^{i} \partial y_{j}^{i}}, \omega\right\rangle_{\lambda},
$$

$$
\frac{\partial I_{\lambda}\left(\sum_{i=1}^{k} \alpha_{i} U_{\mu_{i}, y^{i}}+\omega\right)}{\partial \mu_{j}}=b^{j}\left\langle\frac{\partial^{2} U_{\mu_{j}, y^{j}}}{\partial \mu_{j}^{2}}, \omega\right\rangle_{\lambda}+\sum_{\ell=1}^{N} c_{\ell}^{j}\left\langle\frac{\partial^{2} U_{\mu_{j}, y^{j}}}{\partial \mu_{j} \partial y_{\ell}^{j}}, \omega\right\rangle_{\lambda},
$$

$j=1,2, \ldots, k$.

Before giving the proof of Theorem 1.2, we give the few inequalities that will be needed for our proofs.

By a result of Z.-Q. Wang [27, Lemma 2.3], we have for $N \geq 5, m>1$, $\mu \in \mathbb{R}_{+}$, and $\sigma \in(0,1)$ close to 1 , a constant $C(\sigma)>0$ which is bounded if $\frac{1}{1-\sigma}$ is bounded, such that

$$
\left|\int_{\Omega} U_{\mu, y} \omega\right| \leq\left(1-\frac{\sigma}{2}\right) C(\sigma) \frac{m^{\frac{2}{2-\sigma}}}{\mu^{2}}\|\omega\|_{2^{*}}^{\hat{\sigma}}+\frac{\sigma}{2 m^{2 / \sigma}}\|\omega\|_{2}^{2}
$$

for any $\omega \in H^{1}(\Omega)$, where $\hat{\sigma}=\frac{2(1-\sigma)}{2-\sigma}$.

Direct computation shows that for any $\omega \in E_{\mu, y}^{k}$ and $\mu \in \mathbb{R}_{+}$

$$
\begin{aligned}
& \left|\int_{\Omega} U_{\mu, y}^{2^{*}-1} \omega\right|=\left|\int_{\partial \Omega} \frac{\partial U_{\mu, y}}{\partial \nu} \omega-\lambda \int_{\Omega} U_{\mu, y} \omega\right| \\
& \quad \leq\left(\int_{\partial \Omega}\left|\frac{\partial U_{\mu, y}}{\partial \nu}\right|^{q}\right)^{1 / q}\|\omega\|+\lambda\left|\int_{\Omega} U_{\mu, y} \omega\right| \quad\left(q=\frac{2(N-1)}{N}\right) \\
& \quad=O\left(\frac{1}{\mu^{N / 2}}\right)\|\omega\|+\lambda\left|\int_{\Omega} U_{\mu, y} \omega\right| .
\end{aligned}
$$

From

$$
\left|\frac{\partial U_{\mu, y}}{\partial \mu}\right|=O\left(\frac{1}{\mu}\right) U_{\mu, y},\left|\frac{\partial U_{\mu, y}}{\partial y_{i}}\right|=O\left(\mu U_{\mu, y}\right)
$$


(3.10) and (3.11), we then have

$$
\begin{aligned}
& \left|\int_{\Omega} \frac{\partial U_{\mu, y}}{\partial \mu} \omega\right|=O\left(\frac{1}{\mu^{3}}\right)\|\omega\|_{2^{*}}^{\hat{\sigma}}+O\left(\frac{1}{\mu}\right)\|\omega\|_{2}^{2} \\
& \left|\int_{\Omega} \frac{\partial U_{\mu, y}}{\partial y_{i}} \omega\right|=O\left(\frac{1}{\mu}\right)\|\omega\|_{2^{*}}^{\hat{\sigma}}+O(\mu)\|\omega\|_{2}^{2},
\end{aligned}
$$

where $\hat{\sigma}$ is as in (3.10).

$$
\begin{aligned}
& \left|\int_{\Omega} U_{\mu, y}^{2^{*}-2} \frac{\partial U_{\mu, y}}{\partial \mu} \omega\right| \leq O\left(\frac{1}{\mu^{\frac{N+2}{2}}}\right)\|\omega\|+\lambda\left|\int_{\Omega} \frac{\partial U_{\mu, y}}{\partial \mu} \omega\right| \\
& \left|\int_{\Omega} U_{\mu, y}^{2^{*}-2} \frac{\partial U_{\mu, y}}{\partial y_{i}} \omega\right| \leq O\left(\frac{1}{\mu^{\frac{N-2}{2}}}\right)\|\omega\|+\lambda\left|\int_{\Omega} \frac{\partial U_{\mu, y}}{\partial y_{i}} \omega\right|,
\end{aligned}
$$

for any $\omega \in E_{\mu, y}^{k}$. (3.14) and (3.15) can be established as (3.11).

\section{Proof of Theorem 1.2.}

We argue by contradiction. Suppose (3.1) has a solution $u_{\lambda}$ such that (1.10) holds. It follows from Bahri [5], Rey [19], that for $\lambda$ large

$$
u_{\lambda}=\sum_{i=1}^{k} \alpha_{i, \lambda} U_{\mu_{i, \lambda} y_{\lambda}^{i}}+\omega_{\lambda},
$$

with $\alpha_{i, \lambda}>0, \mu_{i, \lambda}>0, y_{\lambda}^{i} \in \Omega$ and $\omega_{\lambda} \in E_{\mu_{\lambda}, y_{\lambda}}^{k}$, for $\mu_{\lambda}=\left(\mu_{1, \lambda}, \cdots, \mu_{k, \lambda}\right)$, $y_{\lambda}=\left(y_{\lambda}^{1}, \cdots, y_{\lambda}^{k}\right)$, satisfying

$$
\begin{aligned}
& \alpha_{i, \lambda} \rightarrow 1 \quad \text { as } \lambda \rightarrow \infty, \quad i=1, \cdots, k, \\
& \left\|\omega_{\lambda}\right\| \rightarrow 0 \quad \text { as } \lambda \rightarrow \infty, \\
& y_{\lambda}^{i} \rightarrow y^{i} \quad \text { as } \lambda \rightarrow \infty \quad \text { for } i=1, \cdots, k .
\end{aligned}
$$

To simplify our notations, we write $\alpha_{i, \lambda}, y_{\lambda}^{i}$ and $\omega_{\lambda}$ simply as $\alpha_{i}, \mu_{i}, y^{i}$ and $\omega$, respectively, for $i=1, \ldots, k$. By taking $\varphi=\omega$ in (3.7), we have

$$
\begin{aligned}
& \int_{\Omega} \nabla\left(\sum_{i=1}^{k} \alpha_{i} U_{\mu_{i}, y^{i}}+\omega\right) \nabla \omega+\lambda\left(\sum_{i=1}^{k} \alpha_{i} U_{\mu_{i}, y^{i}}+\omega\right) \omega \\
& =\int_{\Omega}\left|\sum_{i=1}^{k} \alpha_{i} U_{\mu_{i}, y^{i}}+\omega\right|^{2^{*}-2}\left(\sum_{i=1}^{k} \alpha_{i} U_{\mu_{i}, y^{i}}+\omega\right) \omega,
\end{aligned}
$$


which gives

$$
\begin{aligned}
& \int_{\Omega}|\nabla \omega|^{2}+\lambda \omega^{2} \\
& =\sum_{i=1}^{k} \alpha_{i}^{2^{*}-1} \int_{\Omega} U_{\mu_{i}, y^{i}}^{2^{*}-1} \omega+\left(2^{*}-1\right) \sum_{i=1}^{k} \alpha_{i}^{2^{*}-2} \int_{\Omega} U_{\mu_{i}, y^{i}}^{2^{*}-2} \omega^{2} \\
& \quad+O\left(\|\omega\|_{2^{*}}^{2+\tilde{\sigma}}\right),
\end{aligned}
$$

for some $\tilde{\sigma} \in\left(0, \min \left\{2^{*}-2,1\right\}\right)$. From (3.10), (3.11), and (3.20), we obtain

$$
\begin{aligned}
& \int_{\Omega}|\nabla \omega|^{2}+\lambda \omega^{2}-\sum_{i=1}^{k} \frac{\lambda \sigma}{2 m^{2 / \sigma}} \alpha_{i} \int_{\Omega} \omega^{2} \\
& -\left(2^{*}-1\right) \sum_{i=1}^{k} \alpha_{i}^{2^{*}-2} \int_{\Omega} U_{\mu_{i}, y^{i}}^{2^{*}-2} \omega^{2} \\
& =O\left(\sum_{i=1}^{k} \frac{\lambda}{\mu_{i}^{2}}\right)\|\omega\|_{2^{*}}^{\widehat{\widehat{\sigma}}}+O\left(\sum_{i=1}^{k} \frac{1}{\mu_{i}^{N / 2}}\right)\|\omega\| .
\end{aligned}
$$

And from a result of Bahri [5], we have, for $\lambda$ large,

$$
\int_{\Omega}|\nabla \omega|^{2}+\frac{1}{2} \lambda \int_{\Omega} \omega^{2}-\left(2^{*}-1\right) \sum_{i=1}^{k} \alpha_{i}^{2^{*}-2} \int_{\Omega} U_{\mu_{i}, y^{i}}^{2^{*}-2} \omega^{2} \geq \rho_{0} \int_{\Omega}|\nabla \omega|^{2},
$$

where $\rho_{0}>0$ is a constant, independent of $\lambda, \mu_{i}, y^{i}, i=1, \ldots, k$.

Taking $m$ suitably large, we obtain from (3.21) and (3.22) that

$$
\int_{\Omega}|\nabla \omega|^{2}+\lambda \omega^{2}=O\left(\sum_{i=1}^{k} \frac{\lambda}{\mu_{i}^{2}}\right)\|\omega\|_{2^{*}}^{\widehat{\sigma}}+O\left(\sum_{i=1}^{k} \frac{1}{\mu_{i}^{N / 2}}\right)\|\omega\|,
$$

which gives

$$
\int_{\Omega}|\nabla \omega|^{2}+\lambda \omega^{2}=O\left(\sum_{i=1}^{k}\left(\frac{\lambda}{\mu_{i}^{2}}\right)^{\frac{2}{2-\widehat{\sigma}}}\right)+O\left(\sum_{i=1}^{k} \frac{1}{\mu_{i}^{N}}\right) .
$$


On the other hand we obtain from (3.6), for each $j=1, \ldots, k$,

$$
\begin{aligned}
& \int_{\Omega} \nabla\left(\sum_{i=1}^{k} \alpha_{i} U_{\mu_{i}, y^{i}}+\omega\right) \nabla U_{\mu_{j}, y^{j}}+\lambda\left(\sum_{i=1}^{k} \alpha_{i} U_{\mu_{i}, y^{i}}+\omega\right) U_{\mu_{j}, y^{j}} \\
= & \int_{\Omega}\left|\sum_{i=1}^{k} \alpha_{i} U_{\mu_{i}, y^{i}}+\omega\right|^{2^{*}-2}\left(\sum_{i=1}^{k} \alpha_{i} U_{\mu_{i}, y^{i}}+\omega\right) U_{\mu_{j}, y^{j}} \\
= & \alpha_{j}^{2^{*}-1} \int_{\Omega} U_{\mu_{j}, y^{j}}^{2^{*}}+\left(2^{*}-1\right) \alpha_{j}^{2^{*}-2} \int_{\Omega} U_{\mu_{j}, y^{j}}^{2^{*}-1} \omega \\
& +\left(2^{*}-1\right) \sum_{\substack{i=1 \\
i \neq j}}^{k} \alpha_{j}^{2^{*}-2} \int_{\omega} U_{\mu_{j}, y^{j}}^{2^{*}-1} U_{\mu_{i}, y^{i}} \\
& +O\left(\sum_{\substack{i=1 \\
i \neq j}}^{k} \int_{\Omega} U_{\mu_{j}, y^{j}}^{2^{*}-1-\theta} U_{\mu_{i}, y^{i}}^{1+\theta}+\int_{\Omega} U_{\mu_{j}, y^{j}}^{2^{*}-1-\theta}|\omega|^{1+\theta}\right)
\end{aligned}
$$

for some $\theta \in\left(0,2^{*}-2\right)$.

Using estimates in Bahri [5] and (3.10), (3.11), we obtain

$$
\begin{aligned}
\alpha_{j}^{2^{*}-1} \int_{\Omega} U_{\mu_{j}, y^{j}}^{2^{*}} & -\alpha_{j} \int_{\Omega}\left|\nabla U_{\mu_{j}, y^{j}}\right|^{2} \\
& =\lambda \sum_{i=1}^{k} \alpha_{i} \int_{\Omega} U_{\mu_{i}, y^{i}} U_{\mu_{j}, y^{j}}+\lambda \sum_{i=1}^{k}\left|\int_{\Omega} U_{\mu_{i}, y^{i}} \omega\right| \\
& +O\left(\frac{1}{\mu_{i}^{\frac{N-2}{2}}}\right)\|\omega\|+O\left(\sum_{\substack{i=1 \\
i \neq j}}^{k} \frac{1}{\mu_{i}^{\frac{N-2}{2}} \mu_{j}^{\frac{N-2}{2}}}\right) \\
& =\lambda \sum_{i=1}^{k} \alpha_{i} \int_{\Omega} U_{\mu_{i}, y^{i}} U_{\mu_{j}, y^{j}}+O\left(\sum_{i=1}^{k}\left(\frac{\lambda}{\mu_{i}^{2}}\right)^{\frac{2}{2-\hat{\sigma}}}\right) \\
& +O\left(\sum_{i=1}^{k} \frac{1}{\mu_{i}^{N-2}}\right) .
\end{aligned}
$$

Notice that, from the definition of $U_{\mu, y}$, there exist two positive constants $D_{1}, D_{2}$, depending on $N$ only, such that

$$
\frac{D_{1}}{\mu^{2}} \leq \int_{\Omega} U_{\mu, y}^{2} \leq \frac{D_{2}}{\mu^{2}}
$$




$$
\int_{\Omega} U_{\mu_{1}, y^{1}} U_{\mu_{2}, y^{2}}=O\left(\frac{1}{\mu_{1}^{\frac{N-2}{2}} \mu_{2}^{\frac{N-2}{2}}}\right) \quad \text { if } y^{1} \neq y^{2},
$$

and from (3.26) we then have

$$
\alpha_{j}-1=\frac{\tilde{D}_{j} \lambda}{\mu_{j}^{2}}+O\left(\sum_{i=1}^{k} \frac{1}{\mu_{i}^{N-2}}\right) \text { for } j=1, \cdots, k,
$$

where $\tilde{D}_{j} \in R^{1}$ satisfies $\frac{1}{2} D_{1} \leq \tilde{D}_{j} \leq 2 D_{2}$. Therefore $\frac{\lambda}{\mu_{j}^{2}} \rightarrow 0$ as $\lambda \rightarrow \infty$, since $\alpha_{j} \rightarrow 1, \mu_{i} \rightarrow \infty$ as $\lambda \rightarrow \infty$.

Next we establish a contradiction. Let us consider the left-hand side of (3.9), which we denote by $L_{j}$, for $j=1, \cdots, k$. We have

$$
\begin{aligned}
& \text { (3.30) } L_{j}=\int_{\Omega} \nabla\left(\sum_{i=1}^{k} \alpha_{i} U_{\mu_{i}, y^{i}}+\omega\right) \nabla \frac{\partial U_{\mu_{j}, y^{j}}}{\partial \mu_{j}} \\
& +\lambda\left(\sum_{i=1}^{k} \alpha_{i} U_{\mu_{i}, y^{i}}+\omega\right) \frac{\partial U_{\mu_{j}, y^{j}}}{\partial \mu_{j}} \\
& -\int_{\Omega}\left|\sum_{i=1}^{k} \alpha_{i} U_{\mu_{i}, y^{i}}+\omega\right|^{2^{*}-2}\left(\sum_{i=1}^{k} \alpha_{i} U_{\mu_{i}, y^{i}}+\omega\right) \frac{\partial U_{\mu_{j}, y^{j}}}{\partial \mu_{j}} \\
& =\sum_{i=1}^{k} \alpha_{i} \int_{\Omega} \nabla U_{\mu_{i}, y^{i}} \nabla \frac{\partial U_{\mu_{j}, y^{j}}}{\partial \mu_{j}}+\lambda \sum_{i=1}^{k} \alpha_{i} \int_{\Omega} U_{\mu_{i}, y^{i}} \frac{\partial U_{\mu_{j}, y^{j}}}{\partial \mu_{j}} \\
& -\alpha_{j}^{2^{*}-1} \int_{\Omega} U_{\mu_{j}, y^{j}}^{2^{*}} \frac{\partial U_{\mu_{j}, y^{j}}}{\partial \mu_{j}} \\
& -\left(2^{*}-1\right) \alpha_{j}^{2^{*}-2} \sum_{\substack{i=1 \\
i \neq j}}^{k} \alpha \int_{\Omega} U_{\mu_{j}, y^{j}}^{2^{*}-} \frac{\partial U_{\mu_{j}, y^{j}}}{\partial \mu_{j}} U_{\mu_{i}, y^{i}} \\
& -\left(2^{*}-1\right) \alpha_{j}^{2^{*}-2} \int_{\Omega} U_{\mu_{j}, y^{j}}^{2^{*}-2} \frac{\partial U_{\mu_{j}, y^{j}}}{\partial \mu_{j}} \omega \\
& +O\left(\frac{1}{\mu_{j}}\right)\|\omega\|_{2^{*}}^{1+\theta}+O\left(\sum_{\substack{i=1 \\
i \neq j}}^{k} \int_{\Omega} U_{\mu_{i}, y^{i}}^{2^{*}-1-\theta} U_{\mu_{j}, y^{j}}^{1+\theta}\right) \frac{1}{\mu_{j}},
\end{aligned}
$$

for some $\theta \in\left(0,2^{*}-2\right)$. 
Using (3.12), (3.14), the estimate in (3.24) for $\omega$, and the estimates in Bahri [5], we obtain

$$
\begin{aligned}
L_{j}= & -\frac{\lambda}{\mu_{j}^{3}} D_{3}+O\left(\frac{\lambda}{\mu_{j}^{\frac{N}{2}}} \sum_{\substack{i=1 \\
i \neq j}}^{k} \frac{1}{\mu_{i}^{\frac{N-2}{2}}}\right)+O\left(\frac{\lambda}{\mu_{j}^{2}}\right)^{\frac{2}{2-\widehat{\sigma}}} \frac{1}{\mu_{j}} \\
& +O\left(\frac{1}{\mu_{j}^{\frac{N}{2}+1}}\right)\|\omega\|+O\left(\frac{1}{\mu_{j}}\right)\|\omega\|_{2^{*}}^{2^{*}-1} \\
& +O\left(\sum_{\substack{i=1 \\
i \neq j}}^{k} \frac{1}{\mu_{i}^{\frac{N-2}{2}}}\right) \\
= & -\frac{\lambda}{\mu_{j}^{3}} D_{3}+O\left(\frac{\lambda}{\mu^{\frac{N}{2}}} \sum_{\substack{i=1 \\
i \neq j}}^{k} \frac{1}{\mu_{i}^{\frac{N-2}{2}}}\right)+O\left(\frac{\lambda}{\mu_{j}^{2}}\right)^{\frac{2}{2-\widehat{\sigma}}} \frac{1}{\mu_{j}} \\
& +O\left(\frac{1}{\mu_{j}^{3}}\right)+O\left(\frac{1}{\mu_{j}} \sum_{i=1}^{k}\left(\frac{\lambda}{\mu_{i}^{2}}\right)^{\left(2^{*}-1\right) \frac{2}{2-\widehat{\sigma}}}\right),
\end{aligned}
$$

where $D_{3}$ is a positive constant.

To estimate the right-hand side of (3.9) for $u_{\lambda}=\sum_{i=1}^{k} \alpha_{i} U_{\mu_{i}, y^{i}}+\omega$, we need first to obtain estimates of $b^{i}, c_{\ell}^{i}$ for $i=1, \cdots, k, \ell=1, \cdots, N$. To this end, we take $\phi=U_{\mu_{j}, y^{j}}, \frac{\partial U_{\mu_{j}, y^{j}}}{\partial \mu_{j}}$ and $\frac{\partial U_{\mu_{j}, y^{j}}}{\partial y_{s}^{j}}$ for $j=1, \cdots, k, s=1, \cdots, N$, respectively, in (3.7). We obtain a system of linear equations satisfied by $a^{i}$, $b^{i}, c_{\ell}^{i}, i=1, \cdots, k, \ell=1, \cdots, N$, as follows:

$$
\begin{aligned}
& \sum_{i=1}^{k} a^{i} \int_{\Omega} \nabla U_{\mu_{i}, y^{i}} \nabla U_{y_{j}, y^{j}}+\sum_{i=1}^{k} b^{i} \int_{\Omega} \nabla \frac{\partial U_{\mu_{i}, y^{i}}}{\partial \mu_{i}} \nabla U_{\mu_{j}, y^{j}} \\
& \quad+\sum_{i=1}^{k} \sum_{\ell=1}^{N} c_{\ell}^{i} \int_{\Omega} \nabla \frac{\partial U_{\mu_{i}, y^{i}}}{\partial y_{\ell}^{i}} \nabla U_{\mu_{j}, y^{j}} \\
& =\frac{\partial I_{\lambda}\left(\sum_{i=1}^{k} \alpha_{i} U_{\mu_{i}, y^{i}}+\omega\right)\left(U_{\mu_{j}, y^{j}}\right)}{\partial \omega}=0, \quad j=1, \ldots, k,
\end{aligned}
$$


$\sum_{i=1}^{k} a^{i} \int_{\Omega} \nabla U_{\mu_{i}, y^{i}} \nabla \frac{\partial U_{\mu_{j}, y^{j}}}{\partial \mu_{j}}+\sum_{i=1}^{k} b^{i} \int_{\Omega} \nabla \frac{\partial U_{\mu_{i}, y^{i}}}{\partial \mu_{i}} \nabla \frac{\partial U_{\mu_{j}, y^{j}}}{\partial \mu_{j}}$

$+\sum_{i=1}^{k} \sum_{\ell=1}^{N} c_{\ell}^{i} \int_{\omega} \nabla \frac{\partial U_{\mu_{i}, y^{i}}}{\partial y_{\ell}^{i}} \nabla \frac{\partial U_{\mu_{j}, y^{j}}}{\partial \mu_{j}}$

$$
=\frac{\partial I_{\lambda}\left(\sum_{i=1}^{k} \alpha_{i} U_{\mu_{i}, y^{i}}+\omega\right)\left(\frac{\partial U_{\mu_{j}, y^{j}}}{\partial \mu_{j}}\right)}{\partial \omega}=\frac{1}{\alpha_{j}} L_{j}, \quad \text { for } j=1, \cdots, k,
$$

(3.34)

$$
\begin{aligned}
& \sum_{i=1}^{k} a^{i} \int_{\Omega} \nabla U_{\mu_{i}, y^{i}} \nabla \frac{\partial U_{\mu_{j}, y^{j}}}{\partial y_{s}^{j}}+\sum_{i=1}^{k} b^{i} \int_{\Omega} \nabla \frac{\partial U_{\mu_{i}, y^{i}}}{\partial \mu_{i}} \nabla \frac{\partial U_{\mu_{j}, y^{j}}}{\partial y_{s}^{j}} \\
& +\sum_{i=1}^{k} \sum_{\ell=1}^{N} c_{\ell}^{i} \int_{\Omega} \nabla \frac{\partial U_{\mu_{i}, y^{i}}}{\partial y_{\ell}^{i}} \nabla \frac{\partial U_{\mu_{j}, y^{j}}}{\partial y_{s}^{j}} \\
= & \frac{\partial I_{\lambda}\left(\sum_{i=1}^{k} \alpha_{i} U_{\mu_{i}, y^{i}}+\omega\right)\left(\frac{\partial U_{\mu_{j}, y^{j}}}{\partial y_{s}^{j}}\right)}{\partial \omega}, \int_{\Omega} \nabla\left(\sum_{i=1}^{k} \alpha_{i} U_{\mu_{i}, y^{i}}+\omega\right) \nabla \frac{\partial U_{\mu_{j}, y^{j}}}{\partial y_{s}^{j}}+\lambda\left(\sum_{i=1}^{k} \alpha_{i} U_{\mu_{i}, y^{i}}+\omega\right) \frac{\partial U_{\mu_{j}, y^{j}}}{\partial y_{s}^{j}} \\
& -\int_{\Omega}\left|\sum_{i=1}^{k} \alpha_{i} U_{\mu_{i}, y^{i}}+\omega\right|^{2^{*}-2}\left(\sum_{i=1}^{k} \alpha_{i} U_{\mu_{i}, y^{i}}+\omega\right) \frac{\partial U_{\mu_{j}, y^{j}}}{\partial y_{s}^{j}} \\
= & \sum_{i=1}^{k} \alpha_{i} \int_{\Omega} \nabla U_{\mu_{i}, y^{i}} \nabla \frac{\partial U_{\mu_{j}, y^{j}}}{\partial y_{s}^{j}}+\lambda \sum_{i=1}^{k} \alpha_{i} \int_{\Omega} U_{\mu_{i}, y^{i}} \frac{\partial U_{\mu_{j}, y^{j}}}{\partial y_{s}^{j}} \\
& -\alpha_{j}^{2^{*}-1} \int_{\Omega} U_{\mu_{j}, y^{j}} \frac{\partial U_{\mu_{j}, y^{j}}}{\partial y_{s}^{j}}
\end{aligned}
$$




$$
\begin{aligned}
& -\left(2^{*}-1\right) \alpha_{j}^{2^{*}-2} \sum_{\substack{i=1 \\
i \neq j}} \int_{\Omega} U_{\mu_{j}, y^{j}}^{2^{*}-2} \frac{\partial U_{\mu_{j}, y^{j}}}{\partial y_{s}^{j}} U_{\mu_{i}, y^{i}} \\
& -\left(2^{*}-1\right) \alpha_{j}^{2^{*}-2} \int_{\Omega} U_{\mu_{j}, y^{j}} \frac{\partial U_{\mu_{j}, y^{j}}}{\partial y_{s}^{j}} \omega \\
& +O\left(\mu_{j}\right)\|\omega\|^{1+\theta}+O\left(\sum_{\substack{i=1 \\
i \neq j}} \int_{\Omega} U_{\mu_{j}, y^{j}}^{2^{*}-\theta} U_{\mu_{i}, y^{i}}^{1+\theta}\right) \mu_{j}
\end{aligned}
$$

(for some $\theta \in\left(0,2^{*}-2\right)$ )

$$
\begin{aligned}
= & O\left(\frac{\lambda}{\mu_{j}}\right)+O\left(\frac{\lambda}{y_{j}^{\frac{N-4}{2}}}\right) \sum_{\substack{i=1 \\
i \neq j}} \frac{1}{\mu_{i}^{\frac{N-2}{2}}}+O\left(\frac{\lambda}{\mu_{j}^{2}}\right)^{\frac{2}{2-\widehat{\sigma}}} \mu_{j} \\
& +O\left(\frac{1}{\mu_{j}}\right)+O\left(\mu_{j}\right) \sum_{i=1}^{k}\left(\frac{\lambda}{\mu_{j}^{2}}\right)^{\frac{2}{2-\widehat{\sigma}}\left(2^{*}-1\right)} \quad, \quad j=1, \cdots, k ; \quad s=1, \cdots, N .
\end{aligned}
$$

From (3.32)-(3.34), and the estimates in Bahri [5], we deduce

$$
\begin{aligned}
b^{j}= & O\left(\frac{\lambda}{\mu_{j}}\right)+O\left(\sum_{i=1}^{k} \frac{1}{\mu_{i}^{\frac{N-2}{2}}}\right) \frac{\lambda}{\mu_{j}^{\frac{N-2}{2}}} \\
& +O\left(\frac{1}{\mu_{j}}\right)+o\left(\sum_{i=1}^{k} \frac{\lambda}{\mu_{i}^{2}}\right), \quad \text { for } j=1, \cdots, k
\end{aligned}
$$

$$
\begin{aligned}
c_{\ell}^{j}= & O\left(\frac{\lambda}{\mu_{j}^{3}}\right)+O\left(\sum_{i=1}^{k} \frac{1}{\mu^{\frac{N+2}{2}}}\right) \frac{\lambda}{\mu^{\frac{N+2}{2}}} \\
& +o\left(\sum_{i=1}^{k} \frac{\lambda}{\mu_{i}^{2}}\right) \frac{1}{\mu_{j}}+O\left(\frac{1}{\mu_{j}^{3}}\right) \\
& \text { for } j=1, \ldots, k, \quad \ell=1, \cdots, N .
\end{aligned}
$$


Substituting (3.35), (3.36) into (3.9), and using (3.31), we obtain

$$
\begin{aligned}
-\frac{\lambda}{\mu_{j}^{3}} D_{3}= & O\left(\frac{\lambda}{\mu_{j}^{\frac{N}{2}}}\right) \sum_{i=1}^{k} \frac{1}{\mu_{i}^{\frac{N-2}{2}}}+O\left(\frac{\lambda}{\mu_{j}^{2}}\right)^{\frac{2}{2-\widehat{\sigma}}} \frac{1}{\mu_{j}}+O\left(\frac{1}{\mu_{j}^{3}}\right) \\
& +O\left(\frac{1}{\mu_{j}}\right) \sum_{i=1}^{k}\left(\frac{\lambda}{\mu_{j}^{2}}\right)^{\left(2^{*}-1\right) \frac{2}{2-\widehat{\sigma}}} \\
= & O\left(\frac{\lambda}{\mu_{j}^{\frac{N}{2}}}\right) \sum_{i=1}^{k} \frac{1}{\mu_{i}^{\frac{N-2}{2}}}+o\left(\frac{\lambda}{\mu_{j}^{3}}\right), \quad j=1, \cdots, k,
\end{aligned}
$$

where $D_{3}$ is a positive constant.

For $N \geq 6$ (3.37) is clearly impossible. For $N=5$, notice that $\frac{\lambda}{\mu_{i}^{2}} \rightarrow 0$ as $\lambda \rightarrow \infty$. Applying the Hölder inequality to the term $\frac{\lambda}{\mu_{j}^{5 / 2}} \frac{1}{\mu_{i}^{3 / 2}}$, we then obtain

$$
-\frac{\lambda}{\mu_{j}^{3}} D_{3}=\sum_{i=1}^{k} o\left(\frac{\lambda}{\mu_{i}^{3}}\right), \quad j=1, \cdots, k .
$$

A contradiction. This completes the proof of Theorem 1.2.

Acknowledgments. After this work was completed, C. Gui told us that he, cooperated with others, has obtained a nonexistence result for $k=1$ (one peaked solutions) when $N=3$ similar to Theorem 1.2 by using a different method.

\section{References}

[1] Adimurthi and G. Mancini, The Neumann problem for elliptic equations with critical nonlinearity, A tribute in honor of G. Prodi, Scuola Norm. Sup. Pisa, (1991), 9-25, MR 94d:35043, Zbl 836.35048.

[2] Adimurthi, G. Mancini and S. L. Yavada, The role of the mean curvature in semilinear Neumann problem involving critical exponent, Comm. Partial Differential Eqns., 20 (1995), 591-631, MR 96b:35058, Zbl 847.35047.

[3] Adimurthi, F. Pacella and S.L. Yavada, Characterization of concentration points and $L^{\infty}$-estimates for solutions of a semilinear Neumann problem involving the critical Sobolev exponent, Diff. Integral Eqns., 8 (1995), 41-68, MR 95f:35076, Zbl 814.35029.

, Interaction between the geometry of the boundary and positive solutions of a semilinear Neumann problem with critical nonlinearity, J. Funct. Anal., 113 (1993), 318-350, MR 94e:35030, Zbl 793.35033. 
[5] A. Bahri, Critical points at infinity in some variational problems, Research Notes in Mathematics, 182, Longman-Pitman, 1989, MR 91h:58022, Zbl 676.58021.

[6] H. Brezis, Nonlinear elliptic equations involving the critical Sobolev exponent, in 'Survey and Perspectives in Directions in P.D.E.', eds. Grandall, Rabinowitz and Turner, New York, Academic Press, (1987), 17-36, Zbl 699.35075.

[7] H. Brezis and L. Nirenberg, Positive solutions of nonlinear elliptic equations involving critical Sobolev exponents, Comm. Pure Appl. Math., 36 (1983), 437-477, MR 84h:35059, Zbl 544.35029.

[8] C. Gui and J. Wei, Multiple interior peak solutions for some singularly perturbed Neumann problem, J. Diff. Equations, 158(1) (1999), 1-27, MR 2000g:35035.

[9] E.F. Keller and L.A. Segel, Initiation of slime mold aggregation viewed as instability, J. Theoret. Bio., 26 (1970), 399-415.

[10] C.S. Lin and W.-M. Ni, On the diffusion coefficient of a semilinear Neumann problem, Calculus of Variations and Partial Differential Equations (S. Hilderbrandt, D. Kinderlehrer, M. Miranda, eds.), Lecture Notes in Math., 1340, Springer-Verlag, (1988), 160-174, MR 90d:35101, Zbl 704.35050.

[11] C.S. Lin, W.-M. Ni and I. Takagi, Large amplitude stationary solutions to a chemotaxis system, J. Diff. Eqns., 72 (1988), 1-27, MR 89e:35075, Zbl 676.35030.

[12] P.L. Lions, The concentration-compactness principle in the calculus of variation, The limiting case, Part 1, Rev. Math. Iberoamen, 1 (1985), 145-201, MR 87c:49007, Zbl 704.49005.

[13] W.-M. Ni and I. Takagi, On the shape of least energy solution to a semilinear Neumann problem, Comm. Pure Appl. Math., 44 (1991), 819-851, MR 92i:35052, Zbl 754.35042.

[14] _ Locating the peaks of least energy solutions to a semilinear Neumann problem, Duke Math. J., 70 (1993), 247-281, MR 94h:35072, Zbl 796.35056.

[15] W.M. Ni, X.B. Pan and I. Takagi, Singular behavior of least energy solutions of a semilinear Neumann problem involving critical Sobolev exponents, Duke Math. J., 67 (1992), 1-20, MR 93j:35081, Zbl 785.35041.

[16] Stanislaus Maier-Paape, Klaus Schmitt and Zhi-Qiang Wang, On Neumann problems for semilinear elliptic equations with critical nonlinearity: Existence and symmetry of multi-peaked solutions, Comm. in Partial Differential Eqns, 22 (1997), 1493-1527, MR 98j:35065, Zbl 895.35040.

[17] R. Palais, The principle of symmetric criticality, Comm. Math. Phys., 69 (1979), 19-30, MR 81c:58026, Zbl 417.58007.

[18] D. Pierotti and S. Terracini, On a Neumann problem with critical exponent and critical nonlinearity on the boundary, Comm. Partial Differential Eqns., 20 (1995), 1043-1075, MR 96c:35060, Zbl 834.35051.

[19] O. Rey, The role of the Green's function in nonlinear elliptic equations involving the critical Sobolev exponent, J. Funct. Anal., 89 (1990), 1-52, MR 91b:35012, Zbl 786.35059.

[20] _ Boundary effect for an elliptic Neumann problem with critical nonlinearity, Comm. Partial Differential Eqns., 22 (1997), 1055-1139, MR 98h:35081, Zbl 891.35040.

[21] _ An elliptic Neumann problem with critical nonlinearity in three dimensional domains, Comm. Contemp. Math., 1 (1999), 405-450, MR 2000i:35067. 
[22] M. Struwe, A global compactness result for elliptic boundary value problems involving limiting nonlinearities, Math. Z., 187 (1984), 511-517, MR 86k:35046, Zbl 545.35034.

[23] X.-J. Wang, Neumann problems of semilinear elliptic equations involving critical Sobolev exponents, J. Diff. Eqns., 93 (1991), 283-310, MR 92j:35072, Zbl 766.35017.

[24] Z.-Q. Wang, The effect of the domain geometry on the number of positive solutions of Neumann problems with critical exponents, Diff. Integral Eqns., 8 (1995), 1533-1554, MR 96g:35018, Zbl 829.35041.

[25] _ Construction of multi-peaked solutions for a nonlinear Neumann problem with critical exponent in symmetric domains, Nonlinear Analysis, TMA, 27 (1996), 1281-1306, MR 97g:35056, Zbl 862.35040.

[26] _ High-energy and multi-peaked solutions for a nonlinear Neumann problem with critical exponents, Proceedings of the Royal Soc. Edinburgh, 125A (1995), 10031029, MR 97a:35075, Zbl 877.35050.

[27] _ Remarks on a nonlinear Neumann problem with critical exponent, Houston J. Math., 20 (1994), 671-84, MR 95m:35028, Zbl 817.35030.

[28] _ On the existence and qualitative properties of solutions for a nonlinear Neumann problem with critical exponent, in 'Proceedings of the First World Congress of Nonlinear Analysts '92 de Gruyter', Lakshmikantham, editor, (1996), 1445-1456, CMP 1389 178, Zbl 864.35041.

[29] _ Nonlinear Neumann problems with critical exponent in symmetric domains, in 'Proceedings of the Conference on Variational Methods in Nonlinear Analysis 1992', A. Ambrosetti, editor, Basel, Gordon \& Breach Publisher, (1995), 205-220, MR 98b:35069, Zbl 844.35037.

[30] J. Wei, On the interior spike layer solutions of singularly perturbed semilinear Neumann problems, Tohoku Math. J., 50 (1998), 159-178, MR 99g:35016, Zbl 918.35024.

[31] S. Yan, On the number of the interior multipeak solutions for singularly perturbed Neumann problems, Topological methods in Nonlinear Anal., 12 (1999), 61-78, MR 01c:35024.

Received September 27, 1999 and revised February 28, 2000. Part of this work was done while Daomin Cao was visiting the School of Mathematics, University of New South Wales as a visiting professor. The first author was supported by 973 project of the Ministry of Sciences and Technology, NSFC and innovation funds of CAS in China. The second and third authors were supported by ARC in Australia.

Institute of Applied Mathematics

ACAdemy of Mathematics and System Sciences

Chinese Academy of Sciences

BEIJING 100080

P.R.CHINA

E-mail address: cao@amath6.amt.ac.cn

School of Mathematics

University of New South Wales

SYDNEY NSW 2052

Australia 
E-mail address: noussair@maths.unsw.edu.au

School of Mathematics and Statistics

UNIVERSITY OF SYDNEY

NSW 2006

Australia

E-mail address: shusen@maths.usyd.edu.au 